\title{
The word entropy and how to compute it
}

\author{
SÉBASTIEN FERENCZI
}

Aix Marseille Université, CNRS, Centrale Marseille, Institut de Mathématiques de Marseille, I2M - UMR 7373 163, avenue de Luminy, 13288 Marseille Cedex 9, France

Christian Mauduit

Institut Universitaire de France

Aix Marseille Université, CNRS, Centrale Marseille, Institut de Mathématiques de Marseille, I2M - UMR 7373 163, avenue de Luminy, 13288 Marseille Cedex 9, France

\author{
Carlos Gustavo Moreira \\ Instituto de Matemática Pura e Aplicada \\ Estrada Dona Castorina 110 \\ 22460-320 Rio de Janeiro, RJ, Brasil
}

\begin{abstract}
The complexity function of an infinite word counts the number of its factors. For any positive function $f$, its exponential rate of growth $E_{0}(f)$ is $\lim _{n \rightarrow \infty} \inf \frac{1}{n} \log f(n)$. We define a new quantity, the word entropy $E_{W}(f)$, as the maximal exponential growth rate of a complexity function smaller than $f$. This is in general smaller than $E_{0}(f)$, and more difficult to compute; we give an algorithm to estimate it. We use $E_{W}(f)$ to compute the Hausdorff dimension of the set of real numbers whose expansions in a given base have complexity bounded by $f$.
\end{abstract}

\section{Definitions}

Let $A$ be the finite alphabet $\{0,1, \ldots, q-1\}$, If $w \in A^{\mathbb{N}}$, and $L(w)$ the set of finite factors of $w$; for any non-negative integer $n$, we write $L_{n}(w)=$ 
$L(w) \cap A^{n}$.

Definition 1.1. The complexity function of $w \in A^{\mathbb{N}}$ is defined for any non-negative integer $n$ by $p_{w}(n)=\left|L_{n}(w)\right|$.

Our work concerns the study of infinite words $w$ the complexity function of which is bounded by a given function $f$ from $\mathbb{N}$ to $\mathbb{R}^{+}$. More precisely, if $f$ is such a function, we put

$$
W(f)=\left\{w \in A^{\mathbb{N}}, p_{w}(n) \leq f(n), \forall n \in \mathbb{N}\right\}
$$

Definition 1.2. If $f$ is a function from $\mathbb{N}$ to $\mathbb{R}^{+}$, we call exponential rate of growth of $f$ the quantity

$$
E_{0}(f)=\lim _{n \rightarrow \infty} \inf \frac{1}{n} \log f(n)
$$

and word entropy of $f$ the quantity

$$
E_{W}(f)=\sup _{w \in W(f)} E_{0}\left(p_{w}\right) .
$$

Of course, if $E_{0}=0$ then $E_{W}$ is zero also. Thus the study of $E_{W}$ is interesting only when $f$ has exponential growth: we are in the little-explored field of word combinatorics in positive entropy, or exponential complexity.

\section{First properties of $E_{0}$ and $E_{W}$}

If $f$ is itself a complexity function (i.e. $f=p_{w}$ for some $w \in A^{\mathbb{N}}$ ), then $E_{W}(f)=E_{0}(f)$. But in general $E_{W}$ may be much smaller than $E_{0}$.

Indeed, for each $1<\theta \leq q$, and $n_{0} \in \mathbb{N}$ such that $\theta^{n_{0}+1}>n_{0}+q-1$, we define the function $f$ by $f(1)=q, f(n)=n+q-1$ for $1 \leq n \leq n_{0}$ and $f(n)=\theta^{n}$ for $n>n_{0}$. We have $E_{0}(f)=\log \theta$ and we can prove that

$$
E_{W}(f) \leq \frac{1}{n_{0}} \log \left(n_{0}+q-1\right)
$$


which can be made arbitrarily small, independently of $\theta$.

We define a regularity condition for $f$.

Definition 2.1. We say that a function $f$ from $\mathbb{N}$ to $\mathbb{R}^{+}$satisfies the conditions $\left(\mathcal{C}^{*}\right)$ if

i) for any $n \in \mathbb{N}$ we have $f(n+1)>f(n) \geq n+1$;

ii) for any $\left(n, n^{\prime}\right) \in \mathbb{N}^{2}$ we have $f\left(n+n^{\prime}\right) \leq f(n) f\left(n^{\prime}\right)$.

But even with $(\mathcal{C} *)$ we may have $E_{W}(f)<E_{0}(f)$. Indeed, let $f$ be the function defined by $f(n)=\left\lceil 3^{n / 2}\right\rceil$ for any $n \in \mathbb{N}$. Then it is easy to check that $f$ satisfies conditions $(\mathcal{C} *)$ and that $E_{0}(f)=\lim _{n \rightarrow \infty} \frac{1}{n} \log f(n)=\log (\sqrt{3})$. On the other hand, we have $f(1)=2, f(2)=3$; thus the language has no 00 or no 11 , and this implies that $E_{W}(f) \leq \log \left(\frac{1+\sqrt{5}}{2}\right)<E_{0}(f)$.

At least, under that condition, we have the important

Theorem 2.2. If $f$ is a function from $\mathbb{N}$ to $\mathbb{R}^{+}$satisfying the conditions $(\mathcal{C} *)$, then $E_{W}(f)>\frac{1}{2} E_{0}(f)$.

We can also show that the constant $\frac{1}{2}$ is optimal.

Finally, it will be useful to know that

Theorem 2.3. For any function $f$ from $\mathbb{N}$ to $\mathbb{R}^{+}$, there exists $w \in W(f)$ such that for any $n \in \mathbb{N}$ we have $p_{w}(n) \geq \exp \left(E_{W}(f) n\right)$.

\section{Algorithm}

In general $E_{W}(f)$ is much more difficult to compute than $E_{0}(f)$; now we will give an algorithm which allows us to estimate with arbitrary precision 
$E_{W}(f)$ from finitely many values of $f$, if we know already $E_{0}(f)$ and have some information on the speed with which this limit is approximated.

We assume that $f$ satisfies conditions $\mathcal{C}^{*}$. We don't loose generality with this assumption, since we may always change a function $f$ which satisfies conditions $\mathcal{C}$ by the function $\tilde{f}$ given recursively by

$$
\tilde{f}(n):=\min \left\{f(n), \min _{1 \leq k<n} \tilde{f}(k) \tilde{f}(n-k)\right\},
$$

which satisfies conditions $\mathcal{C}^{*}$, such that $\tilde{f}(n) \leq f(n), \forall n \in \mathbb{N}$ and $W(\tilde{f})=$ $W(f)$.

Theorem 3.1. There is an algorithm which gives, starting from $f$ and $\varepsilon$, a quantity $h$ such that $(1-\varepsilon) h \leq E_{W}(f) \leq h$. $h$ depends explicitely on $\varepsilon$, $E_{0}(f), N, f(1), \ldots, f(N)$, for an integer $N$ which depends explicitely on $\varepsilon$, $E_{0}(f)$, and an integer $n_{0}$. larger than an explicit function of $\varepsilon$ and $E_{0}(f)$, and such that

$$
\frac{\log f(n)}{n}<\left(1+\frac{E_{0}(f) \varepsilon}{210\left(4+2 E_{0}(f)\right)}\right) E_{0}(f), \quad \text { for } \quad n_{0} \leq n<2 n_{0} .
$$

We shall now give the algorithm. $f$ is given and henceforth we omit to mention it in $E_{0}(f)$ and $E_{W}(f) . \varepsilon \in(0,1)$ is also given.

\section{Description of the algorithm}

Let

$$
\begin{gathered}
\delta:=\frac{E_{0} \varepsilon}{105\left(4+2 E_{0}\right)}<\frac{\varepsilon}{210}, \\
K:=\left\lceil\delta^{-1}\right\rceil+1 .
\end{gathered}
$$

We choose a positive integer

$$
n_{0} \geq K \vee \frac{4 K^{2}}{420^{3} E_{0}}
$$


such that

$$
\frac{\log f(n)}{n}<\left(1+\frac{\delta}{2}\right) E_{0}, \forall n \geq n_{0}
$$

in view of conditions $\mathcal{C}^{*}$, this last condition is equivalent to $\frac{\log f(n)}{n}<(1+$ $\left.\frac{\delta}{2}\right) E_{0}, n_{0} \leq n<2 n_{0}$.

We choose intervals which will be so large that all the lengths of words we manipulate stay in one of them. Namely, for each $t \geq 0$, let

$$
n_{t+1}:=\exp \left(K\left((1+\delta)^{2} E_{0} n_{t}+E_{0}\right)\right) .
$$

We take

$$
N:=n_{K}
$$

We choose now a set $Y \subset A^{N}$ : for a given $Y, L_{n}(Y)=\cup_{\gamma \in Y} L_{(\gamma)}$, we define $q_{n}(Y):=\left|L_{n}(Y)\right|$, for $1 \leq n \leq N$. We look at those $Y$ for which $q_{n}(Y) \leq f(n), \forall n \leq N$, and choose one among them such that

$$
\min _{1 \leq n \leq N} \frac{\log q_{n}(Y)}{n}
$$

is maximum.

Henceforth we omit to mention $Y$ in the $q_{n}(Y)$.

\section{Proposition 3.2.}

$$
\min _{1 \leq n \leq N} \frac{\log q_{n}}{n} \geq E_{W}
$$

Proof. We know by Theorem ?? that there is $\hat{w} \in W(f)$ with $p_{n}(\hat{w}) \geq$ $\exp \left(E_{W} n\right), \forall n \geq 1$. For such a word $\hat{w}$, let $X:=L_{N}(\hat{w}) \subset A^{N}$. We have, for each $n$ with $1 \leq n \leq N, L_{n}(X)=L_{n}(\hat{w})$ and $f(n) \geq \# L_{n}(\hat{w})=p_{n}(\hat{w}) \geq$ $\exp \left(E_{W} n\right)$. Thus $X$ is one of the possible $Y$, and the result follows from the maximality of $\min _{1 \leq n \leq N} \frac{\log q_{n}}{n}$.

On one of the large intervals we have defined, $\frac{\log q_{n}}{n}$ will be almost constant: 
Lemma 3.3. There exists $r<K$, such that

$$
\frac{\log q_{n_{r}}}{n_{r}}<(1+\delta) \frac{\log q_{n_{r+1}}}{q_{n_{r+1}}}
$$

Proof. Otherwise $\frac{\log q_{n_{0}}}{n_{0}} \geq(1+\delta)^{K} \frac{\log q_{n_{K}}}{q_{n_{K}}}$ : as $K>\frac{1}{\delta},(1+\delta)^{K}$ would be close to $e$ for $\delta$ small enough, and is larger than $\frac{9}{4}$ as $\delta<\frac{1}{2}$; thus, as $\frac{\log q_{n_{K}}}{n_{K}} \geq E_{W}$ by Proposition ??, we have $\frac{\log q_{n_{0}}}{n_{0}} \geq \frac{9}{4} E_{W}$, but $q_{n_{0}} \leq f\left(n_{0}\right)$ hence $\frac{\log q_{n_{0}}}{n_{0}}<$ $\left(1+\frac{\delta}{2}\right) E_{0}$, and this contradicts $E_{0} \leq 2 E_{W}$, which is true by Theorem ??.

Let

$$
h:=\frac{\log q_{n_{r}}}{n_{r}}
$$

By Proposition ?? we have

$$
h \geq E_{W} .
$$

What remains to prove is the following proposition (which, understandably, does not use the maximality of $\left.\min _{1 \leq n \leq N} \frac{\log q_{n}}{n}\right)$.

\section{Proposition 3.4.}

$$
(1-\varepsilon) h \leq E_{W}
$$

Proof. Our strategy is to build a word $w$ such that, for all $n \geq 1$,

$$
\exp ((1-\varepsilon) h n) \leq p_{n}(w) \leq f(n)
$$

which gives the conclusion by definition of $E_{W}$. To build the word $w$, we shall define an integer $m$, and build successive subsets of $L_{m}(Y)$; for such a subset $Z$, we order it (lexicographically for example) and define $w(Z)$ to be the Champernowne word on $Z$ : namely, if $Z=\left\{\beta_{1}, \beta_{2}, \ldots, \beta_{t}\right\}$, we build the infinite word

$$
w(Z):=\beta_{1} \beta_{2} \ldots \beta_{t} \beta_{1} \beta_{1} \beta_{1} \beta_{2} \beta_{1} \beta_{3} \ldots \beta_{t-1} \beta_{t} \beta_{1} \beta_{1} \beta_{1} \ldots \beta_{t} \beta_{t} \beta_{t} \ldots
$$


made by concatenation of all words in $Z$ followed by the concatenations of all pairs of words of $Z$ followed by the concatenations of all triples of words of $Z$, etc...

The word $w(Z)$ will satisfy $\exp ((1-\varepsilon) h n) \leq p_{n}(w(Z))$ for all $n$ as soon as

$$
|Z| \geq \exp ((1-\varepsilon) h m)
$$

since, for every positive integer $k$, we will have at least $|Z|^{k}$ factors of length $k m$ in $w(Z)$.

The successive (decreasing) subsets $Z$ of $L_{m}(Y)$ we build will all have cardinality at least $\exp ((1-\varepsilon) h m)$, and the words $w(Z)$ will satisfy $p_{n}(w(Z)) \leq$ $f(n)$ for $n$ in an interval which will increase at each new set $Z$ we build, and ultimately contains all the integers.

We give only the main ideas of the remaining proof. In the first stage we define two lengths of words, $\hat{n}$ and $m>\frac{\hat{n}}{2 \varepsilon}$, which will be both in the interval $\left[n_{r}, n_{r+1}\right]$, and a set $Z_{1}$ of words of length $m$ of the form $\gamma \theta$, for words $\gamma$ of length $\hat{n}$, such that the word $\gamma \theta \gamma$ is in $L_{m+\hat{n}}(Y)$. This is done by looking precisely at twin occurrences of words.

Let $\tilde{\varepsilon}=\frac{\varepsilon}{15}=\frac{7\left(4+2 E_{0}\right) \delta}{E_{0}}>14 \delta$; then we can get such a set $Z_{1}$ with $\left|Z_{1}\right| \geq \exp ((1-\tilde{\varepsilon}) h(m+\hat{n}))$.

In the second stage, we define a a new set $Z_{2} \subset Z_{1}$ in which all the words have the same prefix $\gamma_{1}$ of length $6 \tilde{\varepsilon} h m$, and all the words have the same suffix $\gamma_{2}$ of length $6 \tilde{\varepsilon} h m$, with $\left|Z_{2}\right| \geq\left|Z_{1}\right| \exp (-12 \tilde{\varepsilon} h m-2 \delta h \hat{n})$, and $2 \delta h \hat{n} \leq(1-\tilde{\varepsilon}) \hat{n}$, thus

$$
\left|Z_{2}\right| \geq \exp ((1-13 \tilde{\varepsilon}) h m)
$$

As a consequence of the definition of $Z_{2}$, all words of $Z_{2}$ have the same prefix of length $\hat{n}$, which is a prefix $\gamma_{0}$ of $\gamma_{1}$; as $Z_{2}$ is included in $Z_{1}$, any 
word of $Z_{2}$ is of the form $\gamma_{0} \theta$, amd the word $\gamma_{0} \theta \gamma_{0}$ is in $L_{m+\hat{n}}(Y)$.

At this stage we can prove

Claim 3.5. $p_{w\left(Z_{2}\right)}(n) \leq f(n)$ for all $1 \leq n \leq \hat{n}+1$.

Let us shrink again our set of words.

Lemma 3.6. For a given subset $Z$ of $Z_{2}$, there exists $Z^{\prime} \subset Z,\left|Z^{\prime}\right| \geq(1-$ $\left.\exp \left(-(j-1) \frac{E_{0}}{2}\right)\right)^{j}|Z|$, such that the total number of factors of length $\hat{n}+j$ of all words $\gamma_{0} \theta \gamma_{0}$ such that $\gamma_{0} \theta$ is in $Z^{\prime}$ is at most $f(\hat{n}+j)-j$.

We start from $Z_{2}$ and apply successively Lemma ?? from $j=2$ to $j=6 \tilde{\varepsilon} m$, getting $6 \tilde{\varepsilon} m-1$ succssive sets $Z^{\prime}$; at the end, we get a set $Z_{3}$ such that the total number of factors of length $\hat{n}+j$ of words $\gamma_{0} \theta \gamma_{0}$ for $\gamma_{0} \theta$ in $Z_{3}$ is at most $f(\hat{n}+j)-j$ for $j=2, \ldots, 6 \tilde{\varepsilon} m$, and $\frac{\left|Z_{3}\right|}{\left|Z_{2}\right|}$ is at least

$$
\Pi_{2 \leq j \leq 6 \tilde{\varepsilon} m-\hat{n}}\left(1-\exp \left(-(j-1) \frac{E_{0}}{2}\right)\right)^{j} \geq \Pi_{j \geq 2}\left(1-\exp \left(-(j-1) \frac{E_{0}}{2}\right)\right)^{j},
$$

which implies after computations that

$$
\left.\left|Z_{3}\right| \geq \exp ((1-14 \tilde{\varepsilon}) h m)\right)
$$

We can now bound the number of short factors by using the factors we have just deleted and properties of $\gamma_{0}, \gamma_{1}$ and $\gamma_{2}$.

Claim 3.7. $p_{w\left(Z_{3}\right)}(n) \leq f(n)$ for all $1 \leq n \leq 6 \tilde{\varepsilon} m$.

We shrink our set again.

Let $m \geq n>6 \tilde{\varepsilon} m$; in average a factor of length $n$ of a word in $Z_{3}$ occurs in at most $\frac{m\left|Z_{3}\right|}{f(n)}$ elements of $Z_{3}$. We consider the $\frac{f(n)}{m n^{2}}$ factors of lemgth $n$ which occur the least often. In total, these factors occur in at most $\frac{m\left|Z_{3}\right|}{f(n)} \frac{f(n)}{m n^{2}}=\frac{\left|Z_{3}\right|}{n^{2}}$ elements of $Z_{3}$. We remove these words from $Z_{3}$, for all $m \geq n>6 \tilde{\varepsilon} m$, obtaining a set $Z_{4}$ with $\left|Z_{4}\right| \geq \exp ((1-15 \tilde{\varepsilon}) h m)$. 
We can now control medium length factors, using again the missing factors we have just created, and $\gamma_{1}$ and $\gamma_{2}$, but not $\gamma_{0}$.

Claim 3.8. $p_{w\left(Z_{4}\right)}(n) \leq f(n)$ for all $1 \leq n \leq m$.

Finally we put $Z_{5}=Z_{4}$ if $\left|Z_{4}\right| \leq \exp ((1-4 \tilde{\varepsilon}) h m)$, otherwise we take for $Z_{5}$ any subset of $Z_{4}$ with $\lceil\exp ((1-4 \tilde{\varepsilon}) h m)\rceil$ elements. In both cases we have

$$
\left|Z_{5}\right| \geq \exp ((1-\varepsilon) h m)
$$

For the long factors, we use mainly the fact that there are many missing factors of length $m$, but we need also some help from $\gamma_{1}$ and $\gamma_{2}$.

Claim 3.9. $p_{w\left(Z_{5}\right)}(n) \leq f(n)$ for all $n$.

In view of the considerations at the beginning of the proof of Proposition ??, Claim ?? completes the proof of that proposition, and thus of Theorem $? ?$.

\section{Application}

We define

$$
C(f)=\left\{x=\sum_{n \geq 0} \frac{w_{n}}{q^{n+1}} \in[0,1], w(x)=w_{0} w_{1} \cdots w_{n} \cdots \in W(f)\right\} .
$$

Then we have

Theorem 4.1.

The Hausdorff dimension of $C(f)$ is equal to $E_{W}(f) / \log q$.

\section{References}

[Fal90] K. J. Falconer, Fractal geometry. mathematical foundations and applications John Wiley \& Sons, Chichester 1990. 
[Fer99] S. Ferenczi, Complexity of sequences and dynamical systems, Discrete Math., 206(1-3):145-154, 1999.

[Kůr03] P. Kůrka, Topological and symbolics dynamics, Cours spécialisés SMF,volume 11, 2003.

[MM10] C. Mauduit and C. G. Moreira, Complexity of infinite sequences with zero entropy, Acta Arithmetica 142 (2010), 331-346.

[MM12] C. Mauduit and C. G. Moreira, Generalized Hausdorff dimensions of sets of real numbers with zero entropy expansion, Ergodic Theory and Dynamical Systems 32 (2012), 1073-1089. 